Y. Yoshino

Nagoya Math. J.

Vol. 113 (1989), 89-98

\title{
MODULES WITH LINEAR RESOLUTION OVER A POLYNOMIAL RING IN TWO VARIABLES
}

\author{
YUJI YOSHINO
}

\section{§1. Introduction and main theorem}

Let $k$ be a field and let $S$ be a polynomial ring $k\left[x_{1}, x_{2}, \cdots, x_{n}\right]$ over $k$ in $n$ variables. An $S$-module $M$ is called a module with linear resolution if $M$ has a free resolution;

$$
0 \longrightarrow F_{n} \stackrel{f_{n}}{\longrightarrow} F_{n-1} \longrightarrow \cdots \longrightarrow F_{1} \stackrel{f_{1}}{\longrightarrow} F_{0} \longrightarrow M \longrightarrow 0
$$

where, after taking suitable bases of free modules, all $f_{i}$ 's are matrices consisting of linear forms of $S$. The reader should be referred to EisenbudGoto [2, Sections 0 and 1] for elementary facts concerning modules with linear resolution.

The purpose of this note is to give a complete classification of modules with linear resolution over a polynomial ring in two variables. The main theorem is the following.

THEOREM (1.1). Let $k$ be an algebraically closed field of any characteristic and let $S$ denote a polynomial ring $k[x, y]$ in two variables. Then any finitely generated indecomposable module with linear resolution over $S$ is isomorphic to one of the following;

(i) $S /(x, y) S \simeq k$

(ii) $(x, y)^{n}(n \in \mathbb{N})$ or

(iii) the module $M(n, p)\left(n \in \mathbb{N}, p \in \mathbb{P}_{k}^{1}=k \cup\{\infty\}\right)$, where $M(n, p)$ is given as the cokernel of a linear mapping;

$$
S^{n} \stackrel{A(n, p)}{\longrightarrow} S^{n}
$$

defined by the $n \times n$ matrix

Received April 24, 1987. 


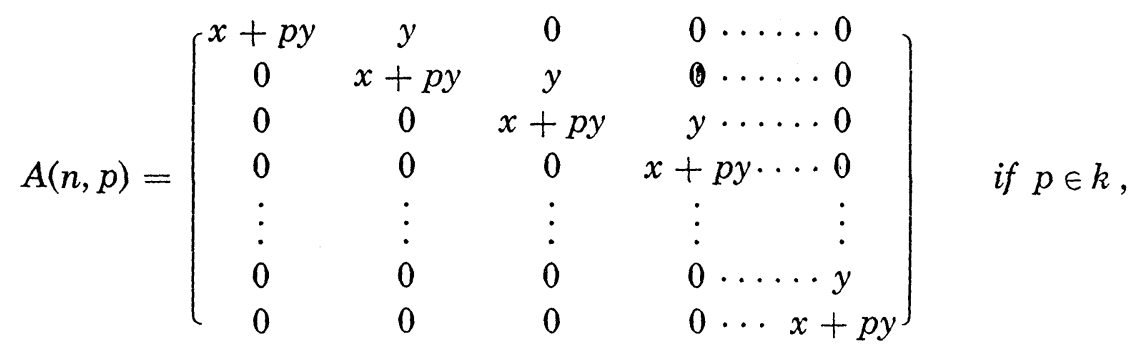

and

$$
\left(\begin{array}{rrrrrr}
y & x & 0 & \cdots & 0 & 0 \\
0 & y & x & \cdots & 0 & 0 \\
0 & 0 & y & \cdots & 0 & 0 \\
\vdots & \vdots & \vdots & & \vdots & \vdots \\
0 & 0 & 0 & \cdots & y & x \\
0 & 0 & 0 & \cdots & 0 & y
\end{array}\right) \quad \text { if } p=\infty
$$

A proof of the theorem will be given in Section 3. To say roughly, it will be shown that the proof is almost equivalent to finding all the indecomposable representations of the euclidean graph of type $\tilde{A}_{1}$. And hence, even if $k$ is not algebraically closed, the theorem will be valid after a slight modification. See Section 3 for more detail. We shall also establish in Section 2 an equivalence between the category of linear complexes over a polynomial ring in $n$ variables and the category of representations of the Grassmann algebra over $n$-dimensional vector space. Using this equivalence, we will be able to classify the modules as in the theorem. Further remarks for the case of three variables will be made in Section 4.

\section{§2. Linear complexes}

In this section $S$ always denotes a polynomial ring $k\left[x_{1}, x_{2}, \cdots, x_{n}\right]$ in $n$ variables over an arbitrary field $k$. Let $V$ be a $k$-vector space of dimension $n$ with a basis $\left\{e_{1}, e_{2}, \cdots, e_{n}\right\}$ and let $V^{*}$ denote the dual space of $V$ with the dual basis $\left\{x_{1}, x_{2}, \cdots, x_{n}\right\}$, that is, $x_{i}\left(e_{j}\right)=\delta_{i j}$ for any $i$ and $j$. We regard $S$ as the symmetric algebra $S\left(V^{*}\right)$ over $V^{*}$.

A linear complex over $S$;

$$
F^{\bullet}: \cdots \longrightarrow F^{p} \stackrel{\varphi_{p}}{\longrightarrow} F^{p+1} \stackrel{\varphi_{p+1}}{\longrightarrow} \cdots \longrightarrow F^{q-1} \stackrel{\varphi_{q-1}}{\longrightarrow} F^{q} \longrightarrow \cdots
$$

is a complex consisting of finite free modules and matrices of linear forms of $S$. Note that a linear complex is completely determined by a set of 
matrices of linear forms $\left\{\varphi_{i}\right\}$ satisfying $\varphi_{i+1} \cdot \varphi_{i}=0$ for all $i$. Let $\left\{\varphi_{i}\right\}$ and $\left\{\psi_{i}\right\}$ give linear complexes, and let $\left\{f_{i}\right\}$ be a morphism of complexes between them, i.e. $f_{i+1} \cdot \varphi_{i}=\psi_{i} \cdot f_{i}$ for all $i$. Then $\left\{f_{i}\right\}$ is said to be a linear morphism if each $f_{i}$ is a matrix consisting of elements in $k=S_{0}\left(V^{*}\right)$. We denote by $\mathscr{L}(S)$ the category of all linear complexes over $S$ and all linear morphisms. We also denote by $\mathscr{L}^{b}(S)$ the full subcategory of $\mathscr{L}(S)$ consisting of all bounded linear complexes. On the other hand, let $G$ be a Grassmann algebra $\Lambda^{*} V$ over $V$ which we always regard as a $\mathbb{Z}$-graded algebra over $k$. The category of all graded left $G$-modules is denoted by $\mathscr{M}_{g r}(G)$ and we denote by $\mathscr{M}_{g r}^{f}(G)$ the full subcategory consisting of all finite modules. We want to establish the following equivalence.

TheOREm (2.1). There is a category equivalence between $\mathscr{L}(S)$ and $\mathscr{M}_{g r}(G)$. And by this equivalence $\mathscr{L}^{b}(S)$ is equivalent to $\mathscr{M}_{g r}^{f}(G)$.

Proof. We define a functor $\Phi: \mathscr{M}_{g r}(G) \rightarrow \mathscr{L}(S)$ as follows. If $M=$ $\sum M_{i}$ is a graded $G$-module, then each $v \in V$ gives a $k$-linear mapping $\varphi_{i}(v): M_{i} \rightarrow M_{i+1}$ for any $i$, where each $\varphi_{i}$ must be linear in $v$ by definition, hence $\varphi_{i}$ 's are matrices consisting of elements in $S_{1}\left(V^{*}\right)$. They satisfy $\varphi_{i+1} \cdot \varphi_{i}=0$ for all $i$, since the action of a square of any $v \in V$ on $M$ is trivial and since each $\varphi_{i+1} \cdot \varphi_{i}$ is a matrix of quadratic forms. Thus the set of matrices $\left\{\varphi_{i}\right\}$ defines a linear complex which we denote by $\Phi(M)$. Note that if $M$ is a finite $G$-module, then $\Phi(M)$ is a bounded complex. Next consider a morphism $f=\sum f_{i}: M=\sum M_{i} \rightarrow N=\sum N_{i}$ in $\mathscr{M}_{g r}(G)$. If $\left\{\varphi_{i}\right\}$ and $\left\{\psi_{i}\right\}$ define respectively $\Phi(M)$ and $\Phi(N)$, then regarding each $f_{i}$ as a matrix consisting of elements in $S_{0}\left(V^{*}\right)=k$, it holds that $f_{i+1} \cdot \varphi_{i}$ $=\psi_{i} \cdot f_{i}$ for all $i$ as matrices over $S$, hence $\left\{f_{i}\right\}$ gives a linear morphism from $\Phi(M)$ to $\Phi(N)$, denoted by $\Phi(f)$.

A functor in the opposite direction will be defined by following the above in reverse. In fact, if $F^{*}: \cdots \rightarrow F^{i} \stackrel{\varphi_{i}}{\longrightarrow} F^{i+1} \stackrel{\varphi_{i+1}}{\longrightarrow} F^{i+2} \rightarrow \cdots$ is a linear complex, then we consider $k$-spaces $M_{i}$ of the same rank as free modules $F^{i}$, and denote $M=\sum M_{i}$ as a $k$-space. We make $M$ a graded $G$-module by defining the action of $v \in V$ on $M_{i}$ as $\varphi_{i}(v)$. We denote by $\Psi\left(F^{\circ}\right)$ the graded $G$-module $M$ and may define a functor $\Psi: \mathscr{L}(S) \rightarrow$ $\mathscr{M}_{g r}(G)$. Then it is easy to see that $\Phi \cdot \Psi=1_{\mathscr{L}(S)}$ and $\Psi \cdot \Phi=1_{\mathscr{k g r}(G)}$, hence the categories are equivalent. It is also obvious that the functors $\Phi$ and $\Psi$ give an equivalence between $\mathscr{L}^{b}(S)$ and $\mathscr{M}_{g r}^{f}(G)$.

Q.E.D.

Remark (2.2). By the equivalence above the grade shifting on modules 
in $\mathscr{M}_{g r}(G)$ corresponds to the degree shifting on complexes. That is, if $M=\sum M_{i}$ is in $\mathscr{M}_{g r}(G)$ and if $F^{*}$ is in $\mathscr{L}(S)$, then the following holds;

$$
\Phi\left(F^{\cdot}[j]\right)=\Phi\left(F^{*}\right)[j] \text { and } \Psi(M[j])=\Psi(M)[j]
$$

where $\left(F^{\cdot}[j]\right)^{i}=F^{j+i}$ and $(M[j])_{i}=M_{j+i}$.

As a corollary of Theorem (2.1) we remark the following fact which, a priori, is non trivial.

Corollary (2.3). The category $\mathscr{L}^{b}(S)$ of linear bounded complexes over $S$ admits the Krull-Schmidt theorem.

\section{§3. Modules over a Grassmann algebra}

By Theorem (2.1) in order to analyze the category $\mathscr{L}(S)$ it will be enough to classify the modules over a Grassmann algebra. If $\operatorname{dim}(V)=1$, it will be easy. In fact the Grassmann algebra $G$ over $V$ is a commutative algebra $k\left[e_{1}\right] /\left(e_{1}^{2}\right)$ and hence $G$ and $k$ are all the indecomposable $G$ modules, correspondingly we may conclude that all the linear complexes over $S=k[x]$ are

$$
0 \longrightarrow S \stackrel{x}{\longrightarrow} S \longrightarrow 0 \text { and } 0 \longrightarrow S \longrightarrow 0 .
$$

However the circumstances are more difficult in the case $S=k[x, y]$. If this is the case, the Grassmann algebra $G$ is a four dimensional $k$ algebra having a basis $\left\{1, e_{1}, e_{2}, e_{1} \wedge e_{2}=-e_{2} \wedge e_{1}\right\}$, and it is easily seen that $G$ is self-injective. Let $R$ be a quotient algebra $G /\left(e_{1} \wedge e_{2}\right)=$ $k\left[e_{1}, e_{2}\right] /\left(e_{1}^{2}, e_{1} e_{2}, e_{2}^{2}\right)$, then the following holds true, and is easy to prove.

Lemma (3.1). If $M$ is an indecomposable left $G$-module which is not free, then $M$ is annihilated by $e_{1} \wedge e_{2}$, i.e. $M$ is a module over $R$.

By this lemma it is sufficient to consider the modules over $R$. We note that the category of modules over $R$ is stably equivalent to the category of representations of the euclidean graph $\tilde{A}_{1}$, hence the classification of those modules are known as a solution to Kronecker's problem. See [1] or [3] for the detail. We exhibit in the following the classification of $R$-modules in the convenient form for us.

LEMMA (3.2). Let $T$ be a commutative $k$-algebra $k\left[e_{1}, e_{2}\right] /\left(e_{1}^{2}, e_{2}^{2}\right)$ where $k$ is an algebraically closed field. Then any finite indecomposable $R$-module is isomorphic to one of the following modules. 
(1) the modules $L_{n}(n \in \mathbb{Z})$ defined as follows; $L_{0}=k$, if $n<0$ then $L_{n}$ is the $(-n)$ th syzygy module of $k$ as a T-module (not as an $R$-module) and if $n>0$ then $L_{n}$ is the T-dual $\operatorname{Hom}_{T}\left(L_{-n}, T\right)$ of $L_{-n}$.

(2) the modules $M(n, p)\left(n \in \mathbb{N}, p \in \mathbb{P}_{k}^{1}=k \cup\{\infty\}\right)$ defined as fcllows; $M(n, p)$ is a graded $R$-module $W_{1} \oplus W_{2}$ having only two graded pieces, where $W=W_{1}=W_{2}$ is an n-dimensional $k$-space, and $e_{1}$ and $e_{2}$ act as mappings of degree 1. If $p \in k$, then $e_{1}$ acts on $W_{1}$ as the identity (to $W_{2}$ ) and $e_{2}$ as a matrix of Jordan canonical form; $\left(\begin{array}{cccccc}p & 1 & 0 & \cdots & 0 & 0 \\ 0 & p & 1 & \cdots & 0 & 0 \\ \vdots & \vdots & \vdots & \vdots & \vdots \\ 0 & 0 & 0 & \cdots & p & 1 \\ 0 & 0 & 0 & \cdots & 0 & p\end{array}\right)$. If $k=\infty$, then $e_{1}$ acts on $W_{1}$ as a matrix; $\left(\begin{array}{cccccc}0 & 1 & 0 & \cdots & 0 & 0 \\ 0 & 0 & 1 & \cdots & 0 & 0 \\ \vdots & \vdots & \vdots & & \vdots & \vdots \\ 0 & 0 & 0 & \cdots & 0 & 1 \\ 0 & 0 & 0 & \cdots & 0 & 0\end{array}\right)$ and $e_{2}$ as the identity.

Remark (3.3). Even if $k$ is not algebraically closed, the lemma above holds true after a slight modification. In fact, it is enough to replace (2) in Lemma (3.2) by the below:

Let $\mathbb{P}_{k}^{1}$ be a set of all monic irreducible polynomials in $k\left[e_{1}\right]$ and a symbol $\infty$. If $p\left(e_{1}\right)=e_{1}^{m}+c_{1} e_{1}^{m-1}+\cdots+c_{m-1} e_{1}+c_{m}$ is an irreducible polynomial, then we denote by $J(p)$ the $m \times m$ matrix;

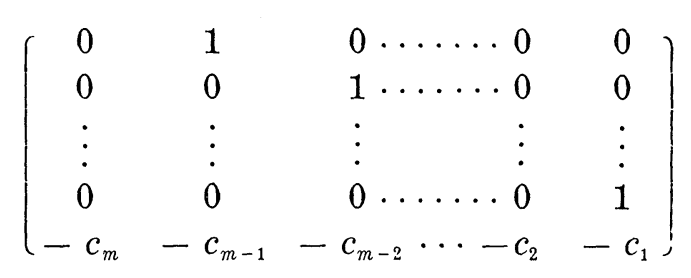

and denote by $I(p)$ the $m \times m$ matrix;

$$
\left(\begin{array}{ccccc}
0 & 0 & \cdots & 0 & 0 \\
0 & 0 & \cdots & 0 & 0 \\
\vdots & \vdots & & \vdots & \vdots \\
0 & 0 & \cdots & 0 & 0 \\
1 & 0 & \cdots & 0 & 0
\end{array}\right)
$$


Then $M(n, p)$ is a graded module $W_{1} \oplus W_{2}$ where $W=W_{1}=W_{2}$ is an $n m$ dimensional space such that if $p \neq \infty$ then $e_{1}$ acts on $W_{1}$ as the identity and $e_{2}$ as a matrix;

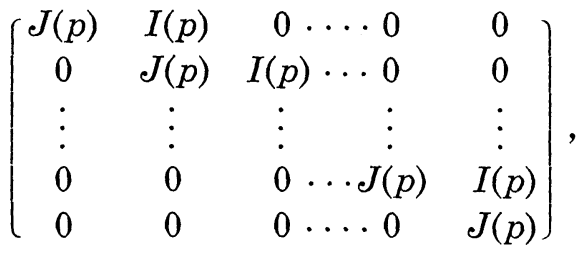

and if $p=\infty$ then $M(n, p)$ is the same as the one in Lemma (3.2).

By Lemma (3.2) and Remark (3.3) above we see that every module over the Grassmann algebra $G$ has a graded structure, and the modules in the list of Lemma (3.2) are thus giving all indecomposable objects in $\mathscr{M}_{g r}^{f}(G)$ up to grade shifting. Combining this with Theorem (2.1) we can classify all the indecomposable bounded linear complexes over $k[x, y]$.

Theorem (3.4). Let $S$ be a polynomial ring $k[x, y]$ over an algebraically closed field $k$. Then any indecomposable bounded linear complex is one of the following:

(1) the Koszul complex; $K^{\cdot}: 0 \rightarrow S \underset{(x,-y)}{\longrightarrow} S^{2} \underset{\left(\begin{array}{l}y \\ x\end{array}\right)}{\longrightarrow} S \rightarrow 0$,

(2) the complexes $L_{n}^{\cdot}(n \in \mathbb{Z}) ; L_{0}^{\cdot}: 0 \rightarrow S \rightarrow 0$, if $n>0$ then

$$
\left(\begin{array}{ccccccc}
y & x & 0 & 0 & \cdots & 0 & 0 \\
0 & y & x & 0 & \ldots & 0 & 0 \\
0 & 0 & y & x & \cdots & 0 & 0 \\
\vdots & \vdots & \vdots & \vdots & & \vdots & \vdots \\
0 & 0 & 0 & 0 & \cdots & x & 0 \\
0 & 0 & 0 & 0 & \ldots & y & x
\end{array}\right)
$$

dual complex $\operatorname{Hom}_{S}\left(L_{-n}^{\cdot}, S\right)$ of $L_{-n}^{\cdot ?}$.

(3) the complexes $M^{\cdot}(n, p)\left(n \in \mathbb{N}, p \in \mathbb{P}_{k}^{1}\right)$; if $p \in k$ then

$$
\begin{aligned}
& M^{*}(n, p): 0 \rightarrow S^{n} \longrightarrow S^{n} \rightarrow 0 \text { and if } p=\infty \text { then }
\end{aligned}
$$

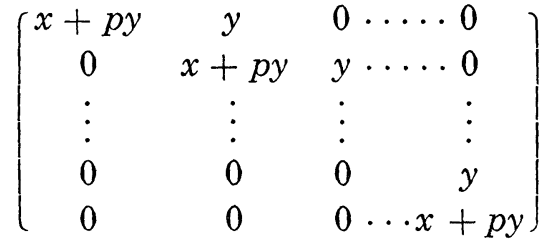




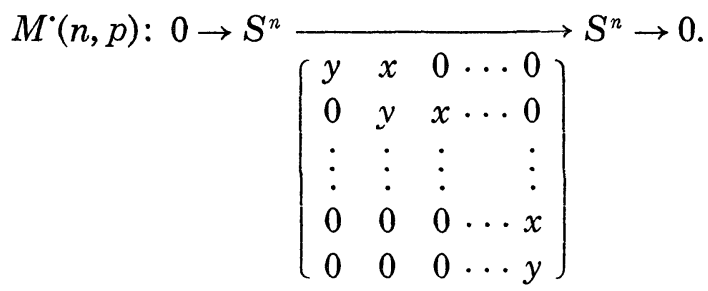

Remark (3.5.) If $k$ is not algebraically closed, then (3) in Theorem (3.4) should read as follows:

Let $\mathbb{T P}_{k}^{1}$ stand for the set of all monic irreducible polynomials in $k\left[e_{1}\right]$ and the symbol $\infty$. The complex $M^{*}(n, \infty)$ is the same as in Theorem (3.4). For a $p \neq \infty$ in $\mathbb{P}_{k}^{1}$, let $I(p)$ and $J(p)$ be as in Remark (3.3). Further we denote by $E(p)$ the identity matrix of the size $\operatorname{deg}(p)$. Then the complex $M^{\cdot}(n, p)(p \neq \infty)$ is the complex;

$$
\begin{aligned}
& 0 \longrightarrow S^{n \cdot \operatorname{deg}(p)} \longrightarrow S^{n \cdot \operatorname{deg}(p)} \longrightarrow 0 .
\end{aligned}
$$

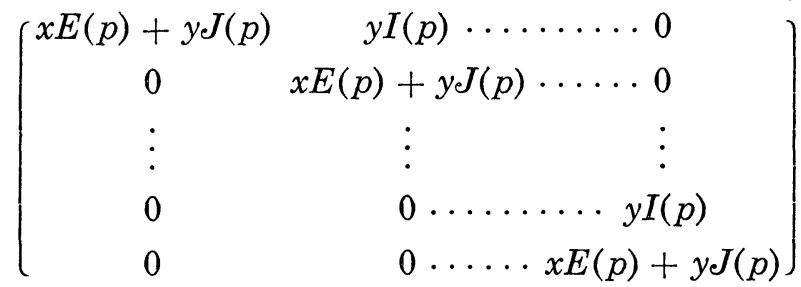

Using Theorem (3.4) we are now able to prove Theorem (1.1). In fact, if $M$ is a finitely generated indecomposable module with linear resolution over $S=k[x, y]$ with $k$ algebraically closed, then the resolution $F^{*}$ of $M$ is an indecomposable linear complex, hence it must be one of the complexes in Theorem (3.2). Since $F^{*}$ is acyclic, it is either $K^{*}, L_{n}^{*}$ $(n \geq 0)$ or $M^{\cdot}(n, p)\left(n \in \mathbb{N}, p \in \mathbb{P}_{k}^{1}\right)$, which gives that $M$ is either $k,(x, y)^{n}$ $(n \geq 0)$ or $M(n, p)$ as in Theorem (1.1).

Q.E.D.

Remark (3.6). Even if $k$ is not algebraically closed, Theorem (1.1) will be valid after replacing $M(n, p)$ in the theorem by the homology module of the complex in Remark (3.5).

\section{§4. Modules with linear resolution over $k[x, y, z]$}

In this section we are concerned with the modules with linear resolution over a polynomial ring $S=k[x, y, z]$ in three variables. By the notation in the beginning of Section 2, $S$ is the symmetric algebra 
over $V^{*}=\langle x, y, z\rangle$ where $V$ denotes the three dimensional $k$ space with a basis $\left\{e_{1}, e_{2}, e_{3}\right\}$ which is dual to $\{x, y, z\}$. We consider a category $\mathscr{T}(S)$ consisting of all finitely generated graded torsion $S$-modules $M$ with linear resolution and with the condition that depth $(M)=2$ (or equivalently $\operatorname{pd}(M)=1)$ and that $M /(x, y) M$ is a direct sum of copies of $k$, and morphisms in $\mathscr{T}(S)$ are graded homomorphisms of degree 0 . For a good evidence that the classification of modules with linear resolution over $S$ would be hopeless, we show that the subcategory $\mathscr{T}(S)$ in the category of all modules with linear resolution is equivalent to the category of all finite modules over the free algebra $k\left\langle e_{1}, e_{2}\right\rangle$ in two variables.

TheOREM (4.1). There is a category equivalence between $\mathscr{T}(S)$ and $\left(k\left\langle e_{1}, e_{2}\right\rangle\right.$-mod.).

Proof. Let $M$ be a module in $\mathscr{T}(S)$. Since $M$ is a torsion module and has depth 2 , it has the free resolution;

$$
0 \longrightarrow S^{n} \stackrel{\varphi}{\longrightarrow} S^{n} \longrightarrow M \longrightarrow 0,
$$

where $\varphi$ is a matrix of linear forms in $S$. Consider the graded module $N$ over the Grassmann algebra $G=\Lambda\left\langle e_{1}, e_{2}, e_{3}\right\rangle$ corresponding to this linear complex under the equivalence of Theorem (2.1). Note that $N$ has only two graded pieces, and we may write $N=W_{1} \oplus W_{2}$, where $W=W_{1}=W_{2}$ is an $n$-dimensional $k$-space. The condition that $M /(x, y) M \simeq k^{n}$ says that, after taking a suitable basis, the matrix $\varphi \otimes S /(x, y) S$ is the identity matrix times $z$, and hence the action of $e_{3}$ on $W_{1}$ to $W_{2}$ is the identity mapping. The actions of $e_{1}$ and $e_{2}$ on $W_{1}=W$ to $W_{2}=W$ define a $k\left\langle e_{1}, e_{2}\right\rangle$-module structure on $W$, which we denote by $\Phi(M)$.

Let $f$ be a graded homomorphism from $M$ to $M^{\prime}$, where $M$ and $M^{\prime}$ are in $\mathscr{T}(S)$. Then $f$ induces a morphism $\left(f_{0}, f_{1}\right)$ between the linear resolutions as follows;

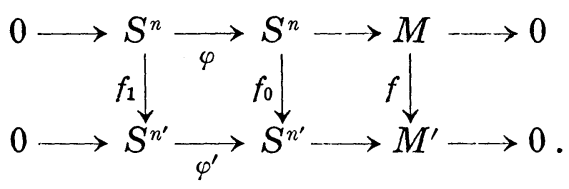

Since $f$ preserves the degree, $\left(f_{0}, f_{1}\right)$ must be a linear morphism. If $N=$ $W_{1} \oplus W_{2}$ (resp. $N^{\prime}=W_{1}^{\prime} \oplus W_{2}^{\prime}$ ) is the graded $G$-module corresponding to $M$ (resp. $M^{\prime}$ ), then $\left(f_{0}, f_{1}\right)$ gives a graded $G$-homomorphism $g=g_{1} \oplus g_{2}$ from $N$ to $N^{\prime}$ by Theorem (2.1). Since the action of $e_{3}$ is the identity on 
$W_{1}$ to $W_{2}$ (resp. on $W_{1}^{\prime}$ to $W_{2}^{\prime}$ ), the diagram; $\left.g_{1}\right|_{W_{1}^{\prime} \underset{i d}{\longrightarrow} W_{2}^{\prime}} ^{W_{2}} W_{2}$ is commutative.

We denote by $\Phi(f)$ the linear mapping $g_{1}: W_{1} \rightarrow W_{1}^{\prime}$ (or equivalently $g_{2}$ : $\left.W_{2} \rightarrow W_{2}^{\prime}\right)$. Since the following diagram is commutative, we see that $\Phi(f)$ is actually a $k\left\langle e_{1}, e_{2}\right\rangle$ homomorphism from $\Phi(M)$ to $\Phi(M)$.

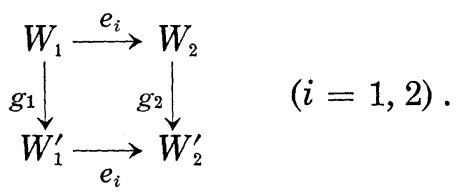

Thus we defined a functor $\Phi$ from $\mathscr{T}(S)$ to $\left(k\left\langle e_{1}, e_{2}\right\rangle\right.$-mod.).

Next we want to define a reverse functor. For this let $W$ be a finite module over $k\left\langle e_{1}, e_{2}\right\rangle$. Then we denote $N=W_{1} \oplus W_{2}$ as a $k$-space where $W=W_{1}=W_{2}$, and define a $G$-module structure by the following;

$e_{i} W_{2}=0 \quad(i=1,2,3), e_{i}: W_{1} \rightarrow W_{2}(i=1$ or 2$)$ is the same as the original action as a $k\left\langle e_{1}, e_{2}\right\rangle$-module, and $e_{3}$ acts on $W_{1}$ to $W_{2}$ as the identity mapping.

Thus we obtain a graded $G$-module $N$, and consider the linear complex corresponding to $N$ under the equivalence in Theorem (2.1), say

$$
0 \longrightarrow S^{n} \stackrel{\varphi}{\longrightarrow} S^{n} \longrightarrow 0 \text {. }
$$

Here that the action of $e_{3}$ is the identity gives that $\varphi \otimes S /(x, y) S$ is a diagonal matrix with diagonal elements $z$. In particular this implies that $\varphi$ is injective and the cokernel $\Psi(W)$ of $\varphi$ is in $\mathscr{T}(S)$. For a $k\left\langle e_{1}, e_{2}\right\rangle-$ homomorphism $h: W \rightarrow W^{\prime}$, we may define a linear mapping $g$ from $N=$ $W_{1} \oplus W_{2}\left(W=W_{1}=W_{2}\right)$ to $N^{\prime}=W_{1}^{\prime} \oplus W_{2}^{\prime}\left(W^{\prime}=W_{1}^{\prime}=W_{2}^{\prime}\right)$ by $g=h \oplus h$. Then it is easy to see that $g$ is, in fact, a $G$-homomorphism, and hence it gives an $S$-homomorphism $\Psi(h)$ from $\Psi(W)$ to $\Psi\left(W^{\prime}\right)$ by Theorem (2.1). This defines the functor $\Psi:\left(k\left\langle e_{1}, e_{2}\right\rangle\right.$-mod. $) \rightarrow \mathscr{T}(S)$.

From the definition of the functors one can easily show that $\Phi \cdot \Psi=$ 1 and $\Psi \cdot \Phi=1$, and this completes the proof.

Q.E.D.

\section{REFERENCES}

[1] Jean Dieudonné, Sur la réduction canonique des couples de matrices, Bull. Soc. Math. Fr., 74 (1946), 130-146. 
[2] David Eisenbud and Shiro Goto, Linear free resolution and minimal multiplicity, Journal of Algebra, 88 (1984), 89-133.

[3] Cornelius Greither, Die unzerlegbaren Moduln über $k[x, y] /\left(x^{2}, y^{2}\right)$, AlgebraBerichte, Seminar F. Kasch, B. Pareigis, Nr. 39, Univ. München, 1979.

Department of Mathematics

Nagoya University

Chikusa-ku, Nagoya 464

Japan 